\title{
Adaptive Swarm Formation Control for Hybrid Ground and Aerial Assets
}

\author{
Laura Barnes ${ }^{1}$, Richard Garcia ${ }^{2}$, MaryAnne Fields ${ }^{2}$ and Kimon Valavanis ${ }^{3}$ \\ ${ }^{1}$ Automation and Robotics Research Institute, University of Texas at Arlington \\ Fort Worth, TX USA \\ ${ }^{2}$ U.S. Army Research Lab \\ Aberdeen Proving Grounds, MD USA \\ ${ }^{3}$ Department of Electrical and Computer Engineering, University of Denver \\ Denver, CO USA
}

\section{Introduction}

The use of Unmanned Aerial Vehicles (UAVs) with Unmanned Ground Vehicles (UGVs) allows for cooperation, coordination, and tight or loose collaboration related to multiple missions. UAVs can provide a global perspective of the surrounding environment, obstacles, and possible threats, broadcasting goals, sub-goals and alterations to the overall mission of the swarm. Further, the deployment of UAVs creates a 3-D sensor network increasing communication capabilities allowing for more complete information about the environment.

UAV-UGV coordination has obvious applicability in military applications due to the line of sight issue. Air vehicles can detect items of interest long before UGVs. Related literature in the area refers to general frameworks and simulation results only. In (Chaimowicz and Kumar 2004; Chaimowicz and Kumar 2004), UGV swarms are coordinated and directed by "shepherd" UAVs. A hierarchy is formed between the UAV and the UGVs. UAVs are responsible for grouping and merging swarms as well as controlling swarm distributions and motion. In (Sukhatme, Montgomery et al. 2001), an architecture is proposed for coordinating an autonomous helicopter and a group of UGVs using decentralized controllers. In (Tanner 2007), an approach is proposed to coordinate groups of ground and aerial vehicles for the purpose of locating a moving target in a given area. This is done by combining decentralized flocking algorithms with navigation functions. Other instances utilizing coordination between air and ground vehicles can be seen in (Elfes, Bergerman et al. 1999; Lacroix, Jung et al. 2001; Stentz, Kelly et al. 2002).

In this work, the problem of controlling and coordinating heterogeneous unmanned systems required to move as a group is addressed. A strategy is proposed to coordinate groups of Unmaned Ground Vehicles with one or more Unmanned Aerial Vehicles (UAVs). UAVs can be utilized in one of two ways: (1) as alpha robots to guide and oversee the UGVs; and (2) as beta robots to surround the UGVs and adapt accordingly. In the first approach, the UAV guides a swarm of UGVs controlling their overall formation. In the second approach, the 
UGVs guide the UAVs controlling their formation. The unmanned systems are brought into a formation utilizing artificial potential fields generated from normal and sigmoid functions. These functions control the overall swarm geometry. Nonlinear limiting functions are defined to provide tighter swarm control by modifying and adjusting a set of control variables forcing the swarm to behave according to set constraints. Formations derived are subsets of elliptical curves but can be generalized to any curvilinear shape. The formation control strategy is a hybrid which can be either completely distributed using only local information summing individually calculated weighted vectors for formation keeping and obstacle avoidance. Moreover, a hiearchical approach with leaders and followers can also be utilized to create a tighter formation and coordinate UAVs and UGVs. The proposed strategy is platform and controller independent as the vector generation is not dependent on the specific robot. Previous research reported in (Barnes, Alvis et al. 2006; Barnes, Fields et al. 2007) presents extensive simulation results and field experiments to validate the formation control methodology.

Both approaches are demonstrated in simulation and experimentally. The first approach is demonstrated experimentally with a fully autonomous UAV for coordination and three UGVs. The autonomous UAV take-off, landing and waypoint navigation is controlled via fuzzy logic controllers. The UGVs utilize identical navigation and formation controllers. To demonstrate the second approach in simulation, a swarm of forty UAVs is utilized in a convoy protection mission. As a convoy of UGVs travels, UAVs dynamically and intelligently adapt their formation in order to protect the convoy of vehicles as it moves. Section 2 discusses the swarm formation controller followed by the UAV controllers in Section 3. Results are presented in Section 4 and 5.

\section{Swarm Formation Controller}

The objective of the formation controller is to attract elements of a swarm into a bounded formation and allow the swarm to stay in that formation as it moves in a mission space. Vector fields and weights are utilized to attract swarm members to the desired surface and keep them distributed about that surface.

\subsection{Generation of Formation Surface}

At any instant in time, the robots can be visualized as particles moving in a potential field generated from a bivariate normal "hill" that controls the velocity and heading of the swarm members. A bivariate normal function with form given in (1):

$$
f(x, y)=e^{-\alpha\left(x-x_{c}\right)^{2}+\gamma^{2}\left(y-y_{c}\right)^{2}}
$$

produces an oval/ellipsoid shaped function. Assuming that the current robot location is at $(x, y)$, the center of the function in (1) is represented by $\left(x_{c}, y_{c}\right)$ in the world reference frame. The 'control' variable $\gamma$ determines the ratio of the minor axis (y-direction) to the major axis (x-direction) affecting the eccentricity of the swarm. The $x$ and $y$ partial derivatives create the velocity vectors that are used to determine the heading and velocity of each member of the swarm as shown in (2): 


$$
\begin{aligned}
& d_{x}=-2 \alpha f(x, y)\left(x-x_{c}\right) \\
& d_{y}=-2 \alpha \gamma f(x, y)\left(y-y_{c}\right)
\end{aligned}
$$

The swarm formation shape has both a local reference and a world reference frame. For the swarm to follow a trajectory in the world reference frame, an axis rotation is required. The heading, $\varphi$, between the swarm formation's $x$-axis and the center $\left(x_{c}, y_{c}\right)$ must be found; the translated and rotated coordinates can be found using (3):

$$
\begin{aligned}
& x_{\text {rot }}=\cos (\phi)\left(x-x_{c}\right)-\sin (\phi)\left(y-y_{c}\right) \\
& y_{\text {rot }}=\sin (\phi)\left(x-x_{c}\right)+\cos (\phi)\left(y-y_{c}\right)
\end{aligned}
$$

The rotated coordinates are then substituted back in to find $d_{x}$ and $d_{y}$.

\subsection{Formation Description}

By attracting swarm members to a specific elliptical ring $R^{*}$ shown in Fig. 1. The swarm can be closely associated with the UAV with the $\left(x_{c}, y_{c}\right)$ denoting its location. For a fixed value of $\gamma$, we will refer to the set of points $(x, y) \in \mathfrak{R}^{2}$ satisfying (4) as the $R^{*}$ ellipse.

$$
R^{* 2}=\left(x-x_{c}\right)^{2} \gamma^{2}\left(y-y_{c}\right)^{2}
$$

A potential field based controller using a small number of physically relevant weights and vectors $v_{i}$ is developed to attract the robots to a neighborhood of the $\mathrm{R}^{*}$ ellipse. This neighborhood is shown in Fig. 1 . The variables $R^{*}-\Delta R_{\text {in }}$ and $R^{*}+\Delta R_{\text {out }}$ denote the inside and outside boundaries of the $R^{*}$ neighborhood respectively as shown in Fig 1 . The desired vector fields will 'trap' the robots in these bands. Typically, this is a very narrow band of allowable space for the robots with a controllable width of $\Delta R_{\text {in }}+\Delta R_{\text {out }}$.

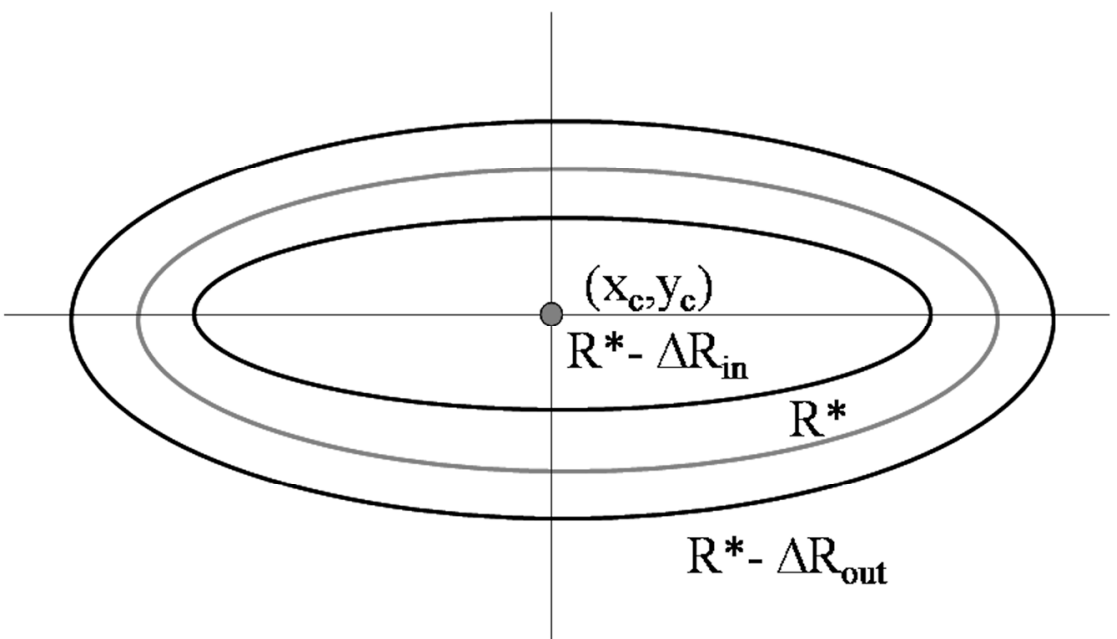

Fig. 1. Elliptical attraction band for the robots 
In the defined vector field, robots with position defined as $r$, starting within the $R^{*}$ ellipse, with:

$$
r=\sqrt{\left(x-x_{c}\right)^{2}+\gamma^{2}\left(y-y_{c}\right)^{2}}
$$

center until they reach the $R^{*}$ neighborhood. Eventually all the robots will be trapped within the neighborhood given in (6):

$$
\left(R^{*}-R_{\text {in }}\right) \leq r \leq\left(R^{*}+R_{\text {out }}\right)
$$

\subsection{Vector Field Generation}

In order to generate the desired vector fields to hold the robots inside the $\mathrm{R}^{*}$ neighborhood, three fields are needed. The gradient vector field, $\mathrm{G}^{-}=-\left(d_{x}, d_{y}\right)$ points away from the center. Vector calculus dictates that the gradient vector field, $\mathrm{G}^{+}=\left(d_{x}, d_{y}\right)$ points in the direction of greatest increase of the function $f(x, y)$, which is towards the center. The vector fields $\left(d_{x},-d_{y}\right)$ and $\left(-d_{x}, d_{y}\right)$ are perpendicular to the gradient $(G \perp)$.

Tighter swarm control is accomplished when restricting the influence of the vector fields to a small region of the $x-y$ plane by multiplying each of the fields by a 'limiting function'. This limiting function controls how far from the center the vectors in the field 'die out' or become smaller than some number $\varepsilon$.

In order to create the desired field, the $\mathrm{G}^{-}$and $\mathrm{G}^{+}$fields must be limited to end at the appropriate boundaries. These fields will be limited with sigmoid functions. The $G^{-}$field

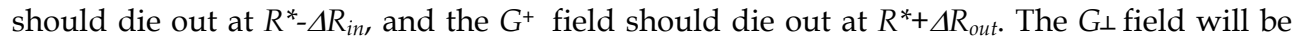
active only inside the elliptical bands so it will die out at $R^{*}-\Delta R_{\text {in }}$ and $R^{*}+\Delta R_{\text {out }}$. This field will be limited with a Normal function.

\subsection{Limiting Functions}

Vector fields 'moving away' from the center (the vectors inside of the ellipse) require a limiting function that approaches zero as the distance from the center is increased; such a limiting function is given in (7):

$$
S_{i n}\left(\alpha_{i n}, r, R_{i n}^{*}, \Delta R_{i n}\right)=1-\frac{1}{1+e^{\alpha_{i n}\left(r-\left(R^{*}-\Delta R_{i n}\right)\right.}}
$$

Gradient vector fields directed towards the center (those vectors outside of the ellipse) are required to approach zero as the vectors 'move towards' the center; this is achieved using the limiting function in (8):

$$
S_{\text {out }}\left(\alpha_{\text {out }}, r, R^{*}, \Delta R_{\text {out }}\right)=1-\frac{1}{1+e^{-\alpha_{\text {out }}\left(r-\left(R^{*}+\Delta R_{\text {out }}\right)\right.}}
$$

Attracting the robot to the $\mathrm{R}^{*}$ neighborhood specified in equation (6) is the first step in the construction of the final vector field. 
An additional vector field can be used to control the robots once they are in the elliptical band. In this field, the robots need to move along the ellipse in a field perpendicular to the previously described gradient fields. A limiting function accomplishing that is given in (9):

$$
N_{\perp}\left(\alpha_{\perp}, r, R^{*}\right)=e^{-\alpha_{\perp}\left(r-R^{*}\right)^{2}}
$$

The plot of the functions $S_{i n}, S_{\text {out }}$, and $N_{\perp}$ as a function of $r$ is provided in Fig. 2. $S_{\text {out }}$ has its largest influence at points whose distance from the center of the ellipse is small. $S_{i n}$ has its greatest influence at points whose distance from the center is large. These functions approach 0 near the $R^{*}$ band. $N_{\perp}$ is only influential with in the ellipsoid bands.

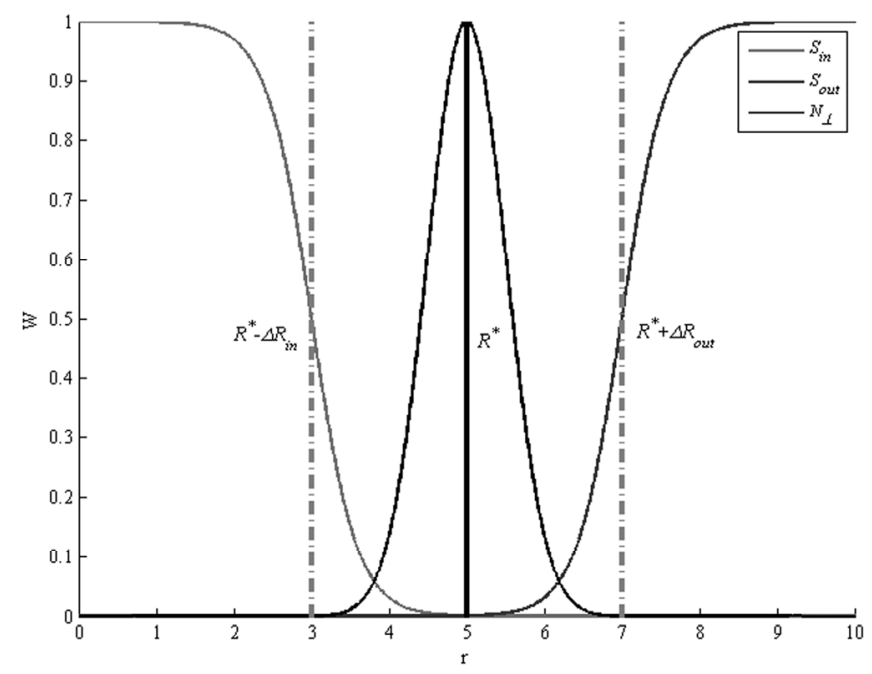

Fig. 2. The weighting functions $S_{\text {in, }}, S_{\text {out }}$, and $N_{\perp}$ as a function of the weighted distance $r$ defined in (5)

In addition, another multiplier to the perpendicular field must be added so the robots do not circle around the ellipse bands. In order for the perpendicular field to change directions, the field perpendicular to the gradient is multiplied by (10) where $y_{\text {rot }}$ is the translated and rotated value of $y$ :

$$
S G N\left(\alpha_{\perp}, y_{r o t}\right)=1-2.0\left(\frac{1}{1+e^{-\alpha_{\perp}\left(y_{r o t}\right)}}\right)
$$

Each of the limiting functions in (7) through (10) contains tuning parameters that may be used as vector field control variables. These functions include one tuning parameter each, which determines how quickly the function approaches zero. 
The parameters $\alpha_{i n}, \alpha_{\text {out }}$, and $\alpha_{\perp}$ control the slope of $S_{\text {in }}(r), S_{\text {out }}(\mathrm{r})$, and $\mathrm{N}_{\perp}(\mathrm{r})$, respectively, for $\mathrm{r}$ in the set $R-\Delta R_{\text {in }}<r<R+\Delta R_{\text {out }}$.

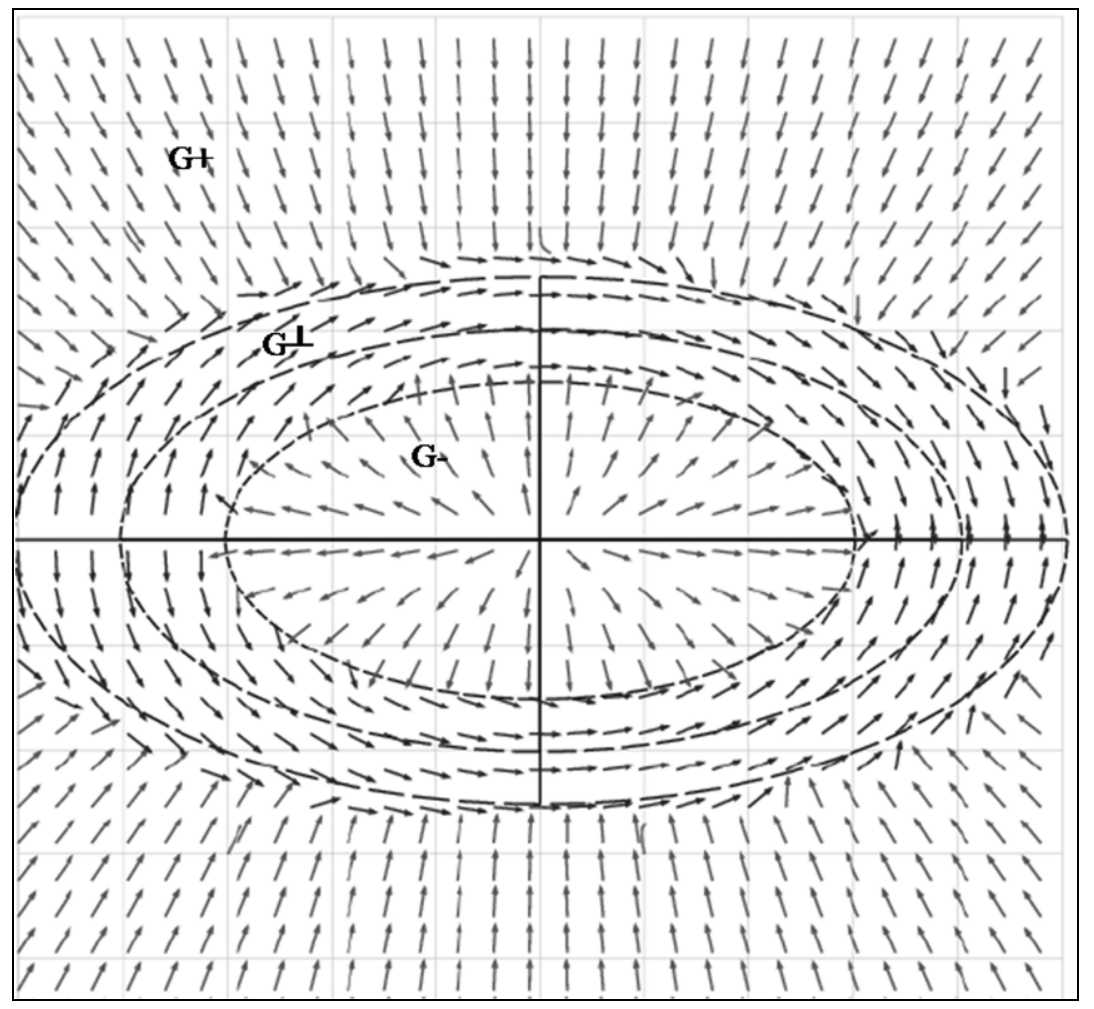

Fig. 3. Final vector field

The value of $S_{i n}\left(R^{*}\right)$ can be made arbitrarily small. Let $\varepsilon>0$ be a small number such that $S_{i n}\left(R^{*}\right)=\varepsilon$. Then the value of $a_{\text {in }}$ can be determined. The same technique is used in the other limiting functions. The resulting equations are shown in (11) to (13):

$$
\begin{gathered}
\alpha_{\text {in }}=\frac{1}{\Delta R_{\text {in }}} \ln \left(\frac{1-\varepsilon}{\varepsilon}\right) \\
\alpha_{\text {out }}=\frac{1}{\Delta R_{\text {out }}} \ln \left(\frac{1-\varepsilon}{\varepsilon}\right) \\
\alpha_{\perp}=\frac{1}{\left(\left(\Delta R_{\text {out }}-\Delta R_{\text {in }}\right) / 2\right)^{2}} \ln (1-\varepsilon)
\end{gathered}
$$


The final vector field is depicted in Fig. 3. Functions $S_{\text {out }}, N_{\perp}$ and $S_{\text {in }}$ impose additional restrictions and constraints on top of and in addition to the initial swarm function $f(x, y)$. The limiting functions, along with vector fields created by the bivariate normal function, may be summed to create swarm movement in formation as a group. When combined, these equations form the velocity and direction of the swarm movement with respect to the center of the swarm, as shown in:

$$
\left[\begin{array}{l}
v_{x} \\
v_{y}
\end{array}\right]=\left(S_{\text {in }}-S_{\text {out }}\right)\left[\begin{array}{l}
d_{x} \\
d_{y}
\end{array}\right]+S G N^{*} N_{\perp}\left[\begin{array}{l}
d_{x} \\
d_{y}
\end{array}\right]_{\perp}
$$

\subsection{Obstacle Avoidance and Swarm Member Distribution}

Vector fields weighted with sigmoid functions may be used for obstacle avoidance as well as controlling member distribution by creating vectors moving away from the center of the obstacle's or other swarm member's location $\left(x_{c o}, y_{c o}\right)$. For the purposes of this work, the concern is formation including distribution of swarm members on the formation. In describing the formation control methodology, it is assumed that the only obstacles are other members of the swarm. The same form of limiting function as $S_{i n}$ may be used. Obstacle avoidance between members is accomplished using Equations (13) to (15):

$$
\begin{gathered}
r_{\text {avoid }}=\sqrt{\left(x-x_{c o}\right)^{2}+\left(y-y_{c o}\right)^{2}} \\
S_{\text {avoid }}\left(\alpha_{\text {avoid }}, r_{\text {avoid }}, \Delta R_{\text {avoid }}\right)=\kappa-\frac{\kappa}{1+e^{\alpha_{\text {avoid }}\left(\sqrt{r_{\text {avoid }}-\Delta R_{\text {avoid }}}\right.}} \\
{\left[\begin{array}{l}
d_{x_{-} \text {avoid }} \\
d_{y_{-} \text {avoid }}
\end{array}\right]=\left[\begin{array}{l}
S_{\text {avoid }}\left(x-x_{c o}\right) \\
S_{\text {avoid }}\left(y-y_{c o}\right)
\end{array}\right]}
\end{gathered}
$$

The weight function generated by a single obstacle is a sigmoid with maximum value $\kappa$ shown in (14). Tuning the parameters $\kappa$ and $a_{\text {avoid }}$ ensures that the collision avoidance field dominates the vector field near an obstacle. Notice that $r_{\text {avoid }}$ is similar to $r$ from Equation (5) except that instead of distance from the center, the distance to the swarm member is used. The $\Delta R_{\text {avoid }}$ parameter denotes the minimum distance from other members. This parameter determines the distribution of swarm members in formation. $S_{\text {out }}$ and $S_{\text {in }}$ get swarm members to the band, but do not control their distribution.

Avoidance of individual swarm members including their distribution is controlled by the range of influence for the avoidance vector field. The $a_{\text {avoid }}$ parameter in (14) controls how quickly vector fields die out near obstacles. As $a_{\text {avoid }}$ decreases, the influence range of the avoidance vector field increases. By controlling the $a_{\text {avoid }}$ parameter, different types of formations can be made within the elliptical bands.

The $a_{\text {avoid }}$ parameter is solved for in the same way as the other sigmoid limiting functions in (12) and (13). The term in (15) is simply summed in (12) to create swarm movement in formation with distribution of swarm members. The $\Delta R_{\text {avoid }}$ parameter specifies the minimum distance between swarm members. Solving for $S_{\text {avoid }}\left(\Delta R_{\text {avoid }}\right)=\varepsilon$ gives: 


$$
\alpha_{\text {avoid }}=\frac{1}{\Delta R_{\text {avoid }}} \ln \left(\frac{\kappa-\varepsilon}{\varepsilon}\right)
$$

\section{UAV Controllers}

The individual UAV helicopters are controlled via four distinct fuzzy controllers. These controllers are responsible for four of the five helicopter inputs: roll, pitch, yaw, and collective. The fifth input, throttle, is output as a constant value throughout the helicopter's navigation routines and thus does not utilize a fuzzy controller. It should be noted that throttle control does vary during the startup and shutdown routines. These routines are simply responsible for starting and stopping the motor during the take-off and landing procedures and utilize a linear throttle increase/decrease to transition the throttle between zero and the constant value used during flight.

The four fuzzy controllers utilized on helicopter are designed using Sugeno constant fuzzy logic and a weighted average defuzzification method. All rules for the controllers are based on the 'and' method and use membership products to determine the strength of each rule. Each controller has a single output which ranges from [-1,1] corresponding to the minimum and maximum Pulse Width (PW) for that particular control respectively. The calculation of the PW from the controller output is done using:

$$
O_{P i}= \begin{cases}\left(\operatorname{Max}_{P_{i}}-N_{P_{i}}\right)^{*} \alpha & \text { for } \alpha \geq 0 \\ \left(N_{P_{i}}-\operatorname{Min}_{P_{i}}\right)^{*} \alpha & \text { for } \alpha<0\end{cases}
$$

where $\operatorname{Max}_{P_{i}}$ is the maximum PW value for servo ' $i$ ', $\operatorname{Min}_{P_{i}}$ is its minimum PW value, $N_{P_{i}}$ is its neutral PW value, $O_{P_{i}}$ is the calculated PW for servo ' $\mathrm{i}$ ', and $\alpha$ is the controller output. $N_{P_{i}}$ is the approximate $\mathrm{PW}$ value of the vehicle in a level hover and is taken from the radio after the vehicle has been properly setup and trimmed by an expert pilot. Note that the helicopter utilizes a three point swashplate which requires cyclic and collective mixing for vehicle control. The method for cyclic and collective mixing is described in great detail in (Garcia 2008).

It should be noted that the control design assumes that the effects of the tail are negligible with respect to roll and pitch control. This assumption is validated by the controller's nonaggressive flight control design, the use of a heading hold gyro, and the minimal tail surface area which creates very little side slip drag. Under this assumption the only real difference between roll and pitch control is the axis of control. As such, the roll and pitch controllers have exactly the same rules with exactly the same outputs and weights. The only difference is the axis used for input and the axis to which the output is applied.

\subsection{Roll / Pitch Controller}

The roll / pitch controller each utilize four inputs, positional error, velocity, orientation angle, and acceleration, each of which is in the local coordinate frame. The positional error, velocity, and orientation inputs each utilize five membership functions with the acceleration input utilizing three membership functions. The fuzzy rules were designed to provide a 
complete set of rules given the inputs, thus the roll and pitch controllers each contain 375 rules, available in (Garcia 2008).

Roll / Pitch, or lateral / longitudinal, control methodology was designed around a hovering technique. The controllers simply attempt to hover at a desired location. Transitions between waypoints are simply an attempt to minimize position error in the hovering location. The fuzzy rule base was designed to first determine a desired input and then compare that to the actual input.

Due to the controllers being designed around a hover routine, the desired positional error is always zero. This value is then compared to the input value. The difference between these values is used to calculate a desired velocity which is consequently compared to the input velocity. The difference between the desired velocity and actual velocity is used to determine a desired orientation. The comparison of the desired orientation is compared to the actual orientation which is utilized to calculate a desired angular rate. This angular rate is then adjusted based on the acceleration input. For example, if the acceleration is currently too high the desired angular rate is decreased. The calcuations referenced here are never truely calculated but describe the mentatiliy used to create the specific fuzzy rules.

\subsection{Collective Controller}

The collective controller utilizes three inputs, positional error, velocity, and acceleration, each of which is in the local coordinate frame. The positional error and velocity inputs each utilize five membership functions with the acceleration input utilizing three membership functions. The fuzzy rules were designed to provide a complete set of rules given the inputs, thus the roll and pitch controllers each contain 75 rules.

The collective control methodology, like the roll / pitch control methodology, was designed around a hovering technique. As such the desired positional error is always zero. This value is then compared to the input positional error. The difference between these values is used to calculate a desired vertical velocity which is consequently compared to the input velocity. The difference is then used to determine a desired acceleration. The difference between the desired and actual acceleration is then used to calculate the control output.

\subsection{Yaw Controller}

The yaw controller utilizes a single input: heading error. The heading error utilizes five membership functions with a single rule dedicated to each. Yaw control is simply based on holding a desired heading. Due to the UAV's use of a heading hold gyro, common on all RC based helicopters, control is calculated by determining a desired angular rate. This rate is calculated by determining the difference between the current heading and the desired heading. The desired rate is then obtained and maintained by the gyro.

The UAV control methodology provides controllers that can be easily modified for desired speeds and orientations. Note that the link between the desired angular rate for roll, pitch, and yaw as well as the vertical acceleration for collective and controller output was hand tuned. Further details into the aspects of the UAV controllers, hardware and software design, as well as experimentation can be seen in (Garcia 2008). 


\section{Application to Convoy Protection Utilizing a UAV Swarm}

In order to demonstrate the proposed approach, it will be applied to the convoy protection problem. Suppose that a swarm of UAVs needs to accompany a convoy of vehicles, surrounding them in a particular formation. In the general case, the convoy can be enclosed in some geometric shape, defined loosely by dimensions, direction of travel, and the center of mass as shown in Fig. 4 . The length of the convoy along the axis of travel is $2 A$. The width of the convoy with respect to the axis of travel is $2 B$.

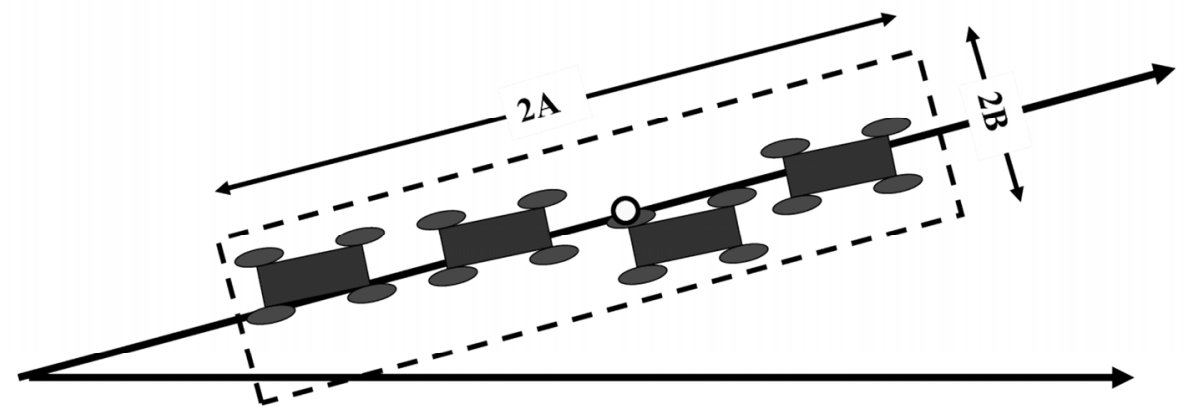

\section{Direction of Travel: $\phi$}

Fig. 4. Convoy description

A field needs to be designed to attract swarm members to surround the convoy in a designated formation. The swarm members need to be close enough to the convoy to offer protection, but far enough to allow the convoy to move safely. The formation controller described in Section 2 is utilized. Assume that the positions of each of the convoy vehicles are known and that the centroid of the convoy is $\left(x_{c}, y_{c}\right)$. It is possible to enclose the convoy within a sequence of concentric ellipses with center $\left(x_{c}, y_{c}\right)$. Fig. 5 depicts three elliptical rings with center $\left(x_{c}, y_{c}\right)$, semi-major axis $A$, and semi-minor axis $B$, surrounding a convoy of vehicles.

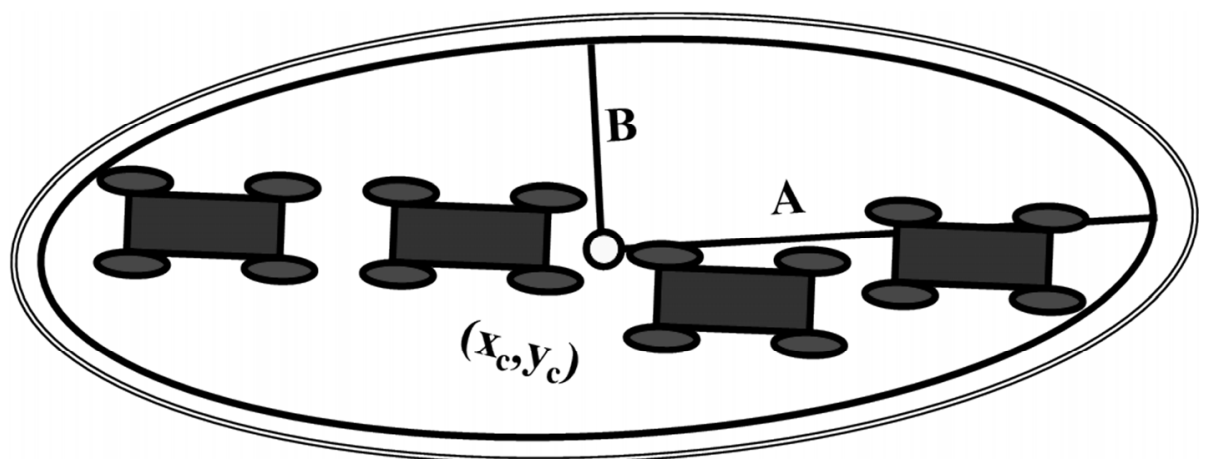

Fig. 5. Convoy of vehicles surrounded by concentric ellipses 
Unreal Tournament is utilized to simulate the real world problem of convoy protection. In this simulation, a convoy of three vehicles is given a set of waypoints on a road and a swarm of forty UAVs is utilized to surround this convoy as it travels. The formation dynamically changes as the convoy travels along the road.

The shape of the elliptical formation is determined by the information provided by the convoy of vehicles that are traveling on the road. They send the swarm parameters describing an ellipse enclosing the convoy - the parameters are the center of the ellipse, the orientation, and the length of the major and minor axes. As the convoy turns the corner, the convoy trucks bunch-up causing the ellipse to become circular. In turn, the swarm redistributes as their elliptical ring becomes circular. This illustrates that proposed approach can easily adapt in differing circumstances.

Fig. 6 shows the swarm formation around the convoy at different time slices. The line is the convoy's path of travel and the darkened circles represent the convoy vehicles. Note that the formation widens and narrows when necessary. This can be noted when the convoy goes around the turn in the road. Fig. 7 shows the parameter values changing over time. Fig. 8 depicts a screenshot from the simulation environment.

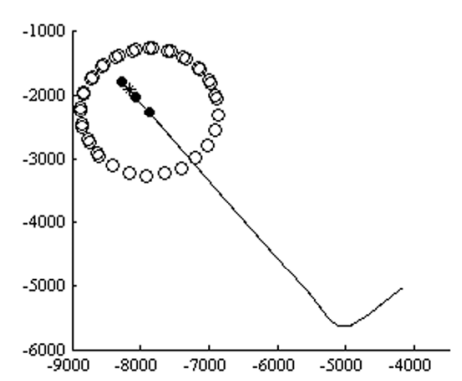

(a)

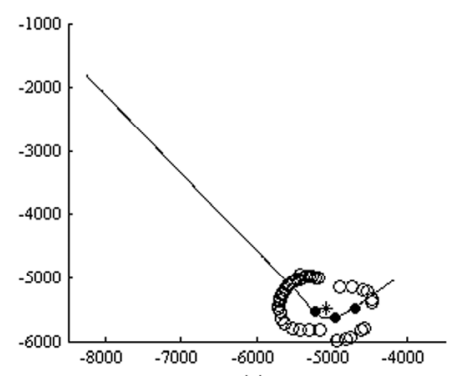

(c)

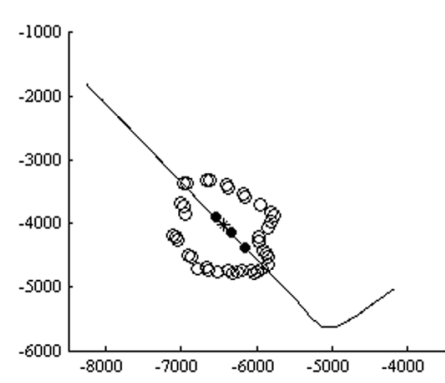

(b)

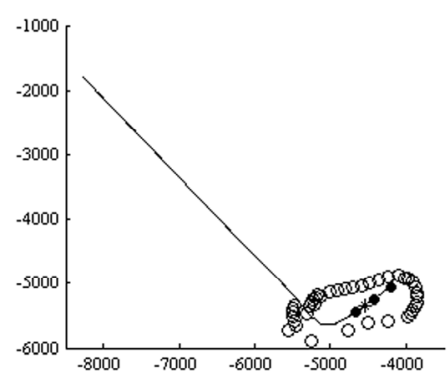

(d)

Fig. 6. Snapshot of a swarm of forty robots travelling and surrounding a convoy of vehicles in formation (a) $t_{1}=1$. (b) $t_{2}=135$. (c) $t_{3}=225$. (d) $t_{4}=260$ 


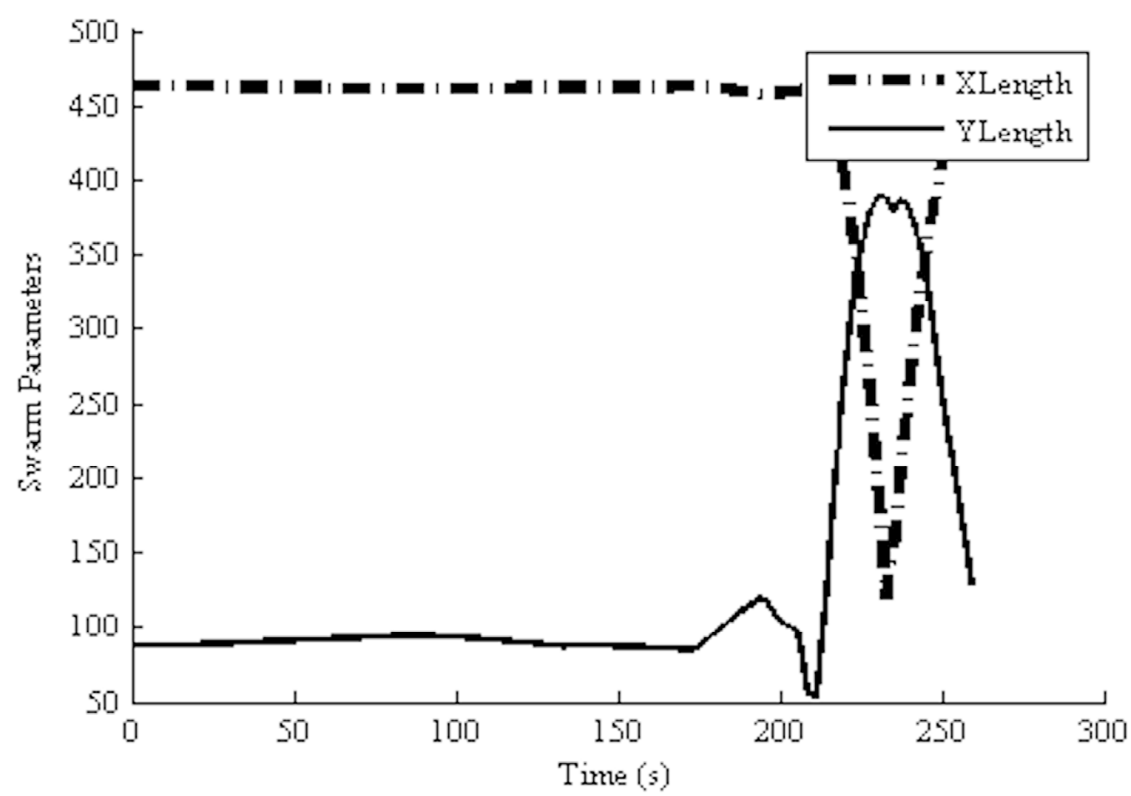

Fig. 7. Swarm formation parameters changing as convoy travels on road network

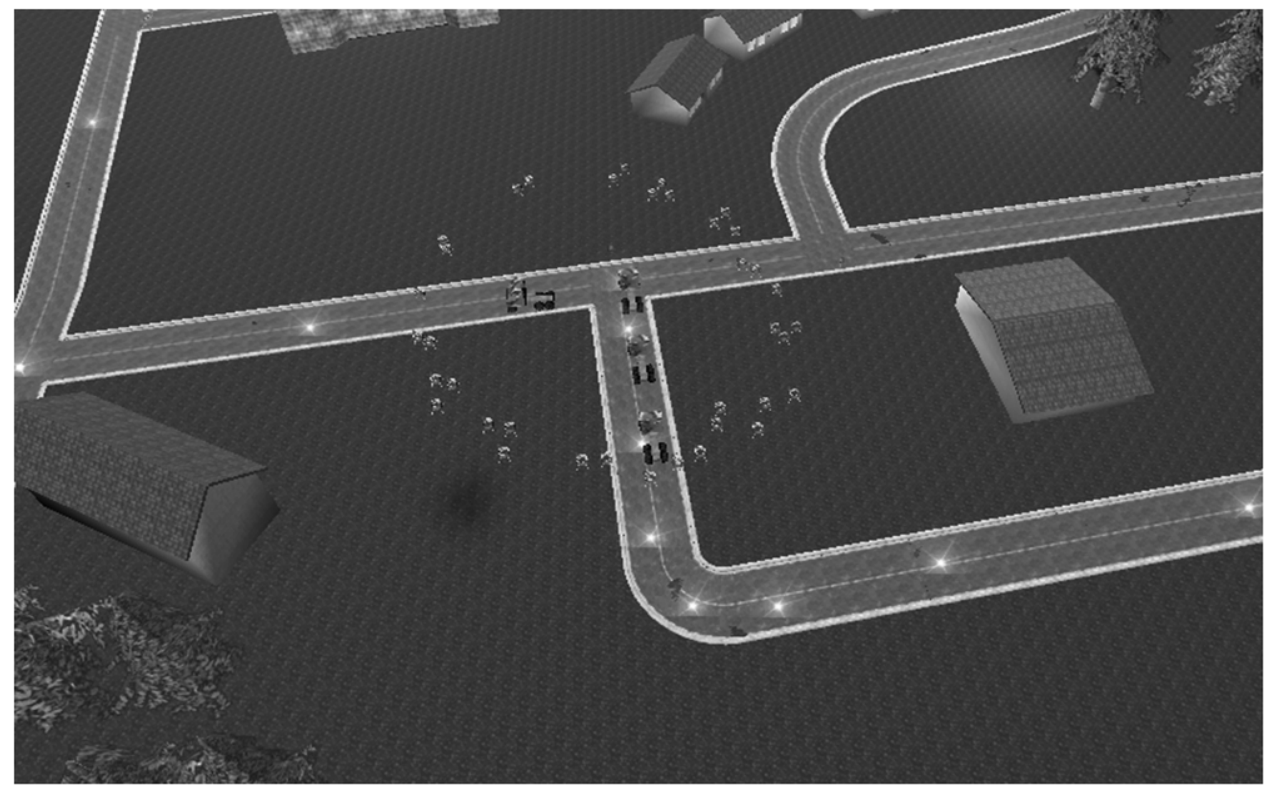

Fig. 8. Convoy protection utilizing a notional UAV swarm 


\section{UAV-UGV Coordination}

In order to describe the coordination between the UAV and the UGV swarm, consider that a swarm of robots needs to accompany an aerial vehicle by surrounding it in a particular formation. A field needs to be designed to attract the swarm members to surround the UAV in a designated formation.

The centroid of the formation is $\left(x_{c}, y_{c}\right)$ or the location of the UAV in two dimensions. The $\mathrm{UAV}$ is surrounded with a sequence of concentric ellipses with the center $\left(x_{c}, y_{c}\right)$. Fig. 9 depicts the envisioned framework. The formation of the ground robots is described by a series of ellipsoids with center $\left(x_{c}, y_{c}\right)$, semi-major axis $2 A$, and semi-minor axis $2 B$, surrounding the UAV.

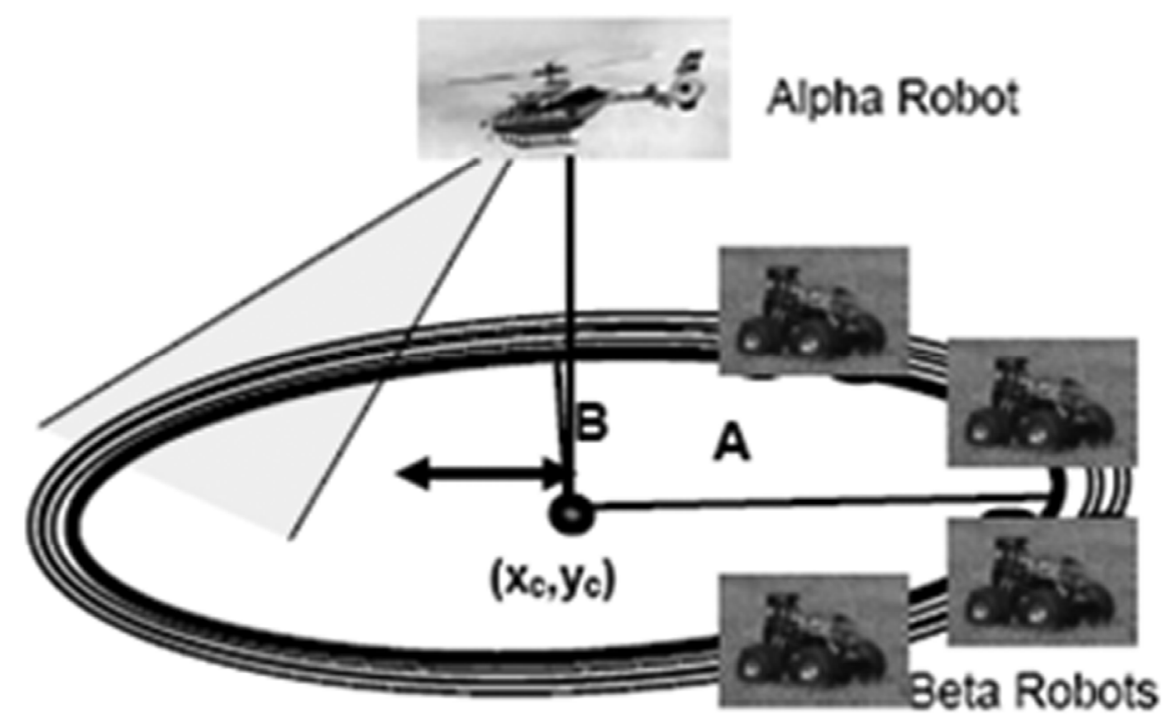

Fig. 9. Framework for UAV-UGV swarm coordination

To validate this work, experiments were performed utilizing an autonomous helicopter as the alpha robot and three custom built RC-trucks as the UGV swarm. The UAV is a Maxi Joker II and the UGVs are Traxxas Emaxx, RC-cars. Both the UAV and UGVs are equipped with a custom built computer system. The UGVs are Ackerman steered and each is equipped with an inertial measurement unit (IMU) and global positioning system (GPS). The UAV is equipped with GPS, IMU, and laser sensors (Garcia and Valavanis 2009). A simple broadcast communication model is used for information relay and exchange between UGVs and UAVs. Fig. 10 depicts the robots and helicopter utilized in these experiments.

In these experiments three UGV vehicles travel in formation surrounding the helicopter. The helicopter, or the alpha robot, acts as the formation center $\left(x_{c}, y_{c}\right)$. The three UGVs (beta robots), surround the UAV and attempt to stay a minimum specified distance away from one another. Table I shows the control parameters used for this experiment. All units are in meters. 
Fig. 11 details the UGV paths with respect to time for a single experiment. The black dotted line represents the path of the UAV. Throughout the mission the UGVs avoid each other while maintaining formation with respect to the UAV's position. Fig. 12 details each of the beta swarm member's distance from the UAV (formation center) over time. The large spikes are due to communication interference which was typical in the experiment field.

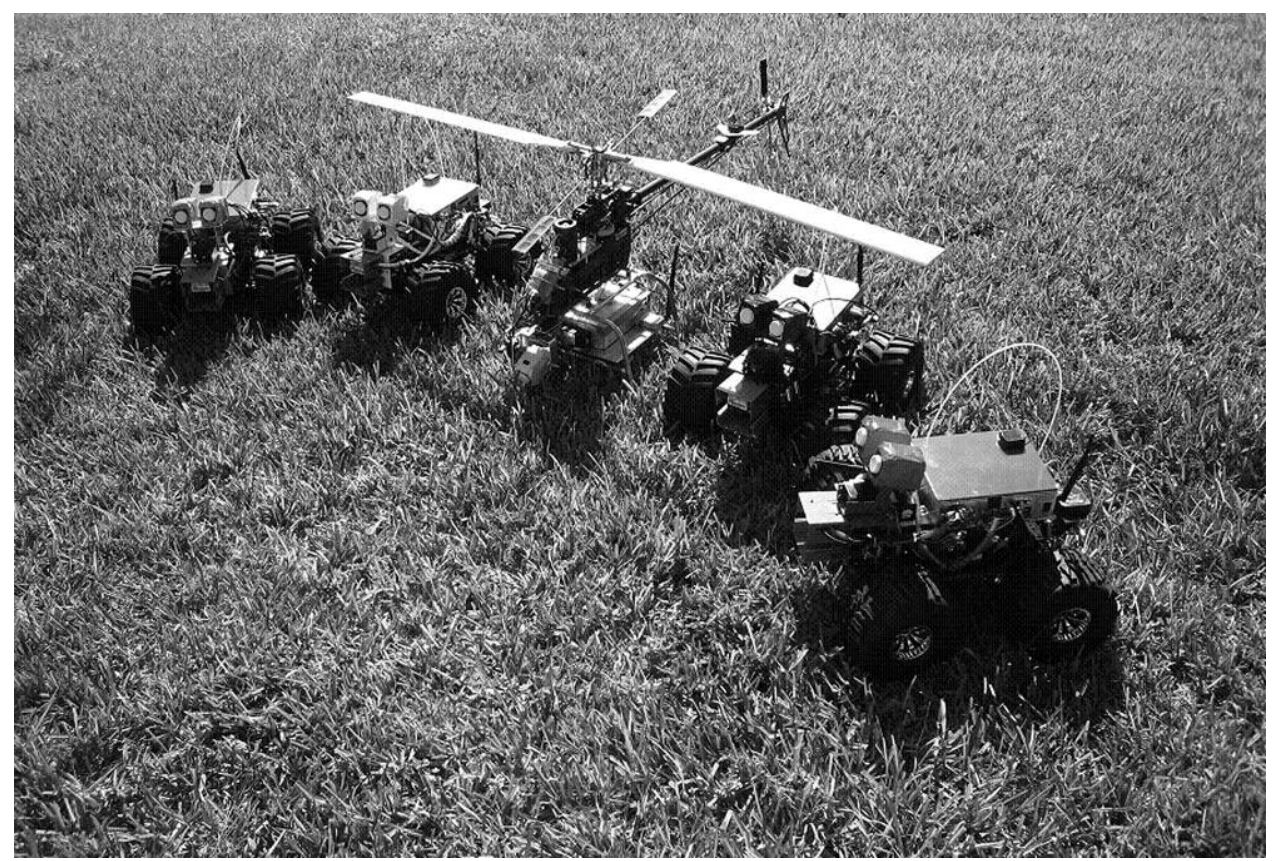

Fig. 10. UAV and UGV robots

\begin{tabular}{|c|l|c|c|c|c|c|c|}
\hline Parameter & $\mathrm{R}^{*}$ & $\gamma$ & $\kappa$ & $\Delta R_{\text {in }}$ & $\Delta R_{\text {out }}$ & $\Delta R_{\text {avoid }}$ & $\varepsilon$ \\
\hline Value & 7 & 0.5 & 1.0 & 3 & 4 & 5 & 0.0001 \\
\hline
\end{tabular}

Table 1. Formation parameters 


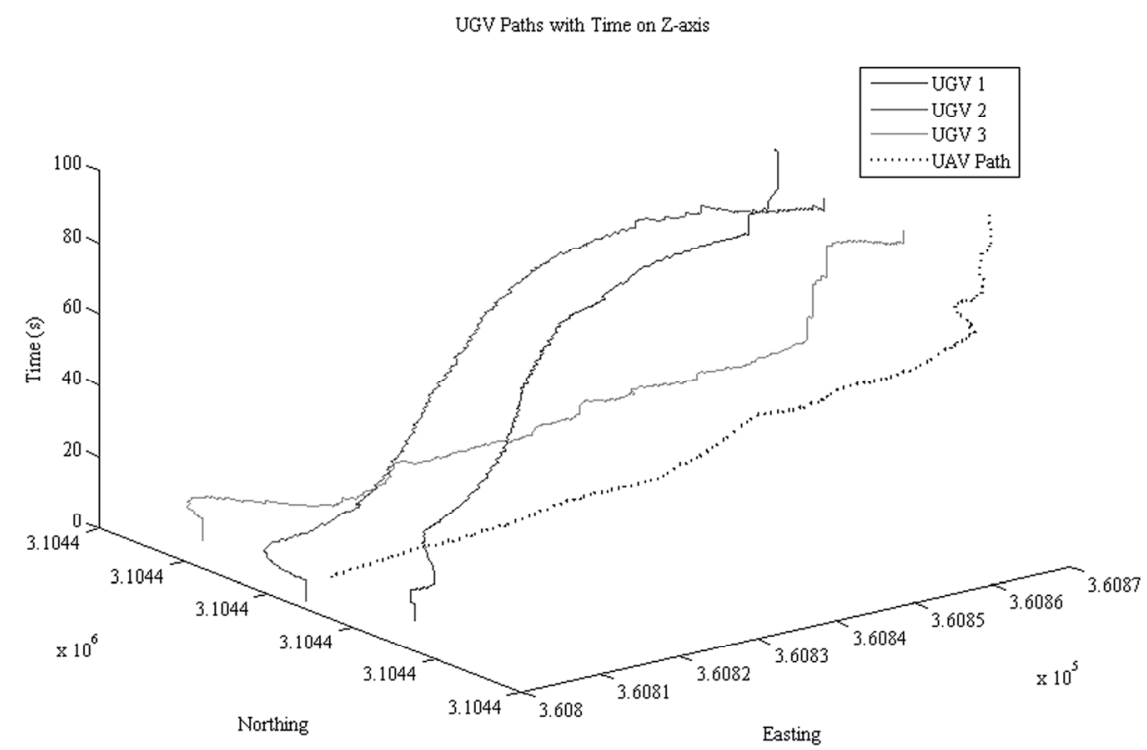

Fig. 11. Robot paths with respect to center $\left(x_{c}, y_{c}\right)$ with time on z-axis

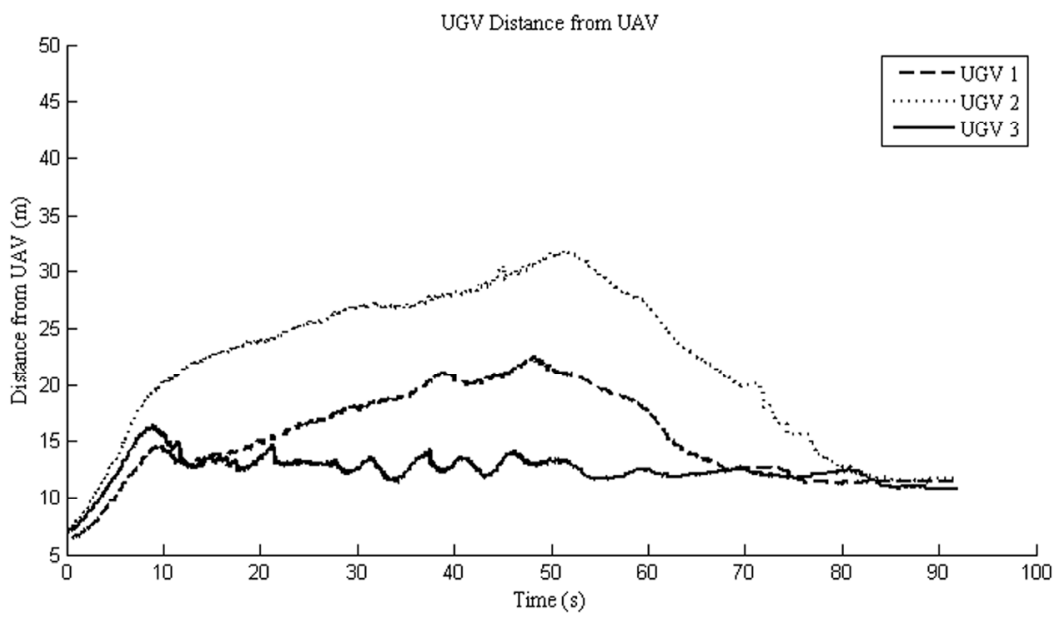

Fig. 12. UGV Distance from UAV

\section{Conclusions}

In this work, a methodology for control and coordination of UAVs and UGVs has been presented. UAVs and UGVs were integrated into a single team and were able to adapt their formation accordingly. Potential field functions together with limiting functions can be successfully utilized to control UGV and UAV swarm formation, obstacle avoidance and the 
overall swarm movement. A single UAV was also successfully used to pull the UGV swarm into formation. These formations can move as a unit, adapt to non-uniform surfaces and change dynamically. In the future, this approach will be expanded to swarms of heterogeneous UAVs utilizing manned and unmanned vehicles as well as swarm adaptation based on environmental parameters. In addition, the variety of formations will be expanded.

\section{References}

Barnes, L., W. Alvis, et al. (2006). Heterogeneous Swarm Formation Control Using Bivariate Normal Functions to Generate Potential Fields. IEEE Workshop on Distributed Intelligent Systems: Collective Intelligence and Its Applications.

Barnes, L., M. A. Fields, et al. (2007). Unmanned Ground Vehicle Swarm Formation Control Using Potential Fields. 15th Mediterranean Conference on Control and Automation.

Chaimowicz, L. and V. Kumar (2004). Aerial Shepherds: Coordination among UAVs and Swarm Robots. International Symposium on Distributed Autonomous Robotic Systems.

Chaimowicz, L. and V. Kumar (2004). A Framework for the Scalable Control of Swarms of Unmanned Ground Vehicles with Unmanned Aerial Vehicles. Proceedings of the International Conference on Robotics and Remote Systems for Hazardous Environments.

Elfes, A., M. Bergerman, et al. (1999). Air-ground robotic ensembles for cooperative applications: concepts and preliminary results. International Conference on Field and Service Robotics.

Garcia, R. D. (2008). Designing an Autonomous Helicopter Testbed: From Conception through Implementation. Computer Science and Engineering. Tampa, University of South Florida.

Garcia, R. D. and K. P. Valavanis (2009). "The Implementation of an Autonomous Helicopter Testbed." J. Intell. Robotics Syst. 54(1-3): 423-454.

Lacroix, S., I.-K. Jung, et al. (2001). Towards cooperative air/ground robotics: issues related to environment modeling. International Conference on Advanced Robotics.

Stentz, A., A. Kelly, et al. (2002). Integrated air/ground vehicle system for semi-autonomous off-road navigation. AUVSI Unmanned Systems Symposium.

Sukhatme, G., J. Montgomery, et al. (2001). Experiments with aerial-ground robots. Robot Teams: From Diversity to Polymorphism, AK Peters.

Tanner, H. G. (2007). Switched UAV-UGV Cooperation Scheme for Target Detection. IEEE International Conference on Robotics and Automation. 


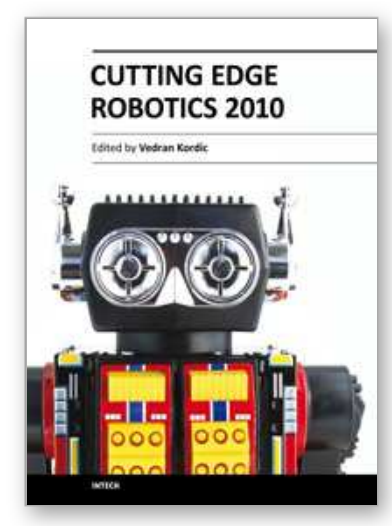

\author{
Cutting Edge Robotics 2010 \\ Edited by Vedran Kordic
}

ISBN 978-953-307-062-9

Hard cover, 440 pages

Publisher InTech

Published online 01, September, 2010

Published in print edition September, 2010

Robotics research, especially mobile robotics is a young field. Its roots include many engineering and scientific disciplines from mechanical, electrical and electronics engineering to computer, cognitive and social sciences. Each of this parent fields is exciting in its own way and has its share in different books. This book is a result of inspirations and contributions from many researchers worldwide. It presents a collection of a wide range of research results in robotics scientific community. We hope you will enjoy reading the book as much as we have enjoyed bringing it together for you.

\title{
How to reference
}

In order to correctly reference this scholarly work, feel free to copy and paste the following:

Laura Barnes, Richard Garcia, Mary Anne Fields and Kimon Valavanis (2010). Adaptive Swarm Formation Control for Hybrid Ground and Aerial Assets, Cutting Edge Robotics 2010, Vedran Kordic (Ed.), ISBN: 978953-307-062-9, InTech, Available from: http://www.intechopen.com/books/cutting-edge-robotics2010/adaptive-swarm-formation-control-for-hybrid-ground-and-aerial-assets

\section{INTECH}

open science | open minds

\section{InTech Europe}

University Campus STeP Ri

Slavka Krautzeka 83/A

51000 Rijeka, Croatia

Phone: +385 (51) 770447

Fax: +385 (51) 686166

www.intechopen.com

\section{InTech China}

Unit 405, Office Block, Hotel Equatorial Shanghai

No.65, Yan An Road (West), Shanghai, 200040, China

中国上海市延安西路65号上海国际贵都大饭店办公楼 405 单元

Phone: +86-21-62489820

Fax: +86-21-62489821 
(C) 2010 The Author(s). Licensee IntechOpen. This chapter is distributed under the terms of the Creative Commons Attribution-NonCommercialShareAlike-3.0 License, which permits use, distribution and reproduction for non-commercial purposes, provided the original is properly cited and derivative works building on this content are distributed under the same license. 